This Section of Epidemiology and Psychiatric Sciences appears in each issue of the Journal to stress the role of the epidemiological approach to promote advances in the field of clinical psychopharmacology, with a particular attention to controversial findings. The ultimate aims are to help develop a more critical attitude towards the results of research studies published in the international literature, to promote original research projects with higher methodological standards, and to implement the most relevant results of research in every-day clinical practice. These contributions are written in house by the journal's editorial team or commissioned by the Section Editor (no more than 1000 words, short unstructured abstract, four key-words, one Table or Figure and up to ten references).

\title{
Autism spectrum disorders: weighing the risk of SSRI exposure in pregnancy
}

\author{
G. Ostuzzi and C. Barbui* \\ Department of Public Health and Community Medicine, Section of Psychiatry, University of Verona, Verona, Italy
}

A possible link between prenatal exposure to the selective serotonin reuptake inhibitors (SSRIs) and development of autism spectrum disorders (ASDs), previously suggested by two case-control studies, was not confirmed by a recent cohort study that followed for 5-10 years more than 600,000 births. However, this study failed to demonstrate that SSRI exposure during pregnancy is safe in terms of child development outcomes, as an increased risk of ASDs cannot be completely ruled out. In the present article, the main strengths and weaknesses of this study are briefly analysed, including a possibility of confounding by indication.

Received 17 March 2014; Accepted 18 March 2014; First published online 1 May 2014

Key words: Autism spectrum disorders, maternal depression, pregnancy, selective serotonin reuptake inhibitors..

Recent epidemiological evidence has consistently shown a high prevalence of depression in women during pregnancy. Maternal depression represents a major public health challenge, as it has potentially harmful implications for mothers and children (Grigoriadis et al. 2013). Antidepressants, particularly the selective serotonin reuptake inhibitors (SSRIs), are recommended in women during pregnancy, considering their relatively safe profile in terms teratogenic effects, delivery complications and neonatal toxicity, with the potential exception of paroxetine and with some methodological concerns on the overall reliability of these data (Ross et al. 2013).

* Address for correspondence: Professor Corrado Barbui, Department of Public Health and Community Medicine, Section of Psychiatry, University of Verona, Piazzale L.A. Scuro, 10 - 37134 Verona, Italy.

(Email: corrado.barbui@univr.it)
While the majority of studies are focused on teratogenic effects, delivery complications and neonatal toxicity associated with SSRI use, current knowledge on the potential harmful effects of the SSRIs on child development is very unsatisfactory. Studies tend to focus on perinatal outcomes measured immediately after birth, whilst complex neurodevelopmental disorders, such as autism spectrum disorders (ASDs) or other neuro-psychiatric disorders, may require several years to develop; methodologically, this implies designing studies with very long-term follow-up assessments. Recently, two casecontrol studies were able to investigate the adverse effects of SSRI exposure on child development (Croen et al. 2011; Rai et al. 2013). A significant association between prenatal exposure to SSRIs and development of ASDs was found. These findings are potentially consistent with the theory of developmental neurotransmitters dysregulation in ASDs (Neuhaus et al. 2010). 
In order to further explore this association, a research team from Denmark recently performed a large population- and register-based prospective cohort study (Hviid et al. 2013). The joint use of a number of national registries (including the Medical Birth Registry, the National Prescription Registry and several others) allowed us to collect and combine many demographics and clinical information, including data on SSRIs exposure before and during pregnancy, and diagnosis of ASDs in children. Researchers included only singleton births in a 10-year period, and excluded conditions associated with an increased risk of ASDs (e.g., congenital rubella syndrome or some genetic disorders in parents). Information on a number of potential confounders was additionally collected (e.g., maternal psychiatric diagnosis, age at the onset of pregnancy, parity, etc.). Children were prospectively followed from birth until 5-10 years of age.

Out of a total study cohort of 626875 live births, 6068 children $(1.0 \%)$ were exposed to SSRIs during pregnancy. In terms of risk of developing ASD, the study found that, in comparison with no SSRI exposure, children of women exposed to SSRIs both before and during pregnancy were more likely to receive a diagnosis of ASD according to a crude analysis (rate ratio 1.62; 95\% confidence interval [CI], 1.23-2.13). However, this significant association disappeared after adjusting for psychiatric diagnosis and other confounders (rate ratio $1.20 ; 95 \%$ CI $0.90-1.61$ ). Similarly, in comparison with no SSRI exposure, children of women exposed only during pregnancy were at increased risk in the crude (rate ratio 1.79, 95\% CI 1.19-2.69) but not in the adjusted (rate ratio 1.40, 95\% CI 0.92-2.13) analysis.

In the present observational study, considering that the reference category was constituted by women with no SSRI exposure, confounding by indication might be expected to be a major issue (Barbui et al. 2013). This bias occurs when the indication for the intervention (maternal depression) is itself a risk factor for the illness (ASDs in children) (Salas et al. 1999). This is probably the case in this study, as epidemiological data suggested that maternal depression might indeed be a risk factor for developing ASD (Rezendes \& Scarpa, 2011; Rai et al. 2013). This might explain the finding that significant associations disappeared after adjusting for psychiatric diagnosis and, intriguingly, that in comparison with no SSRI exposure, children of women exposed to SSRIs only before pregnancy (which could be considered as a proxy of suffering from depression during pregnancy) were at increased risk of developing ASD (rate ratio 1.46, 95\% CI 1.171.81).

Despite maternal depression was included in the analysis as a confounding variable, information on maternal diagnoses were available only from psychiatric hospitals and psychiatric units, but not from primary care settings, where the majority of cases of maternal depression is expected to be recognised. It is therefore possible that residual confounding by indication might have occurred. A second concern refers to the study cohort. Although it is representative of the Denmark population, it may not be representative of other populations, as shown by the prevalence of pregnancy-related use of SSRIs, which was very low in the present study $(0.97 \%)$, as compared, for example, with the USA (5.6\%). The same applies to the prevalence of autism, lower in this study $(0.62 \%)$ as compared with, for example, the USA $(1.14 \%)$. A third reason for concern is the exposure variable, as the present study employed SSRI prescription as a proxy of SSRI use. The general issue that a relevant proportion of medicines prescribed for people with chronic conditions are not taken might be particularly challenging in this study, as depressed women might decide not to take a prescribed treatment with SSRIs, when pregnant, more often than depressed women who are not pregnant. A final concern is that length of follow-up was 5-10 years, which means that some children were followed for 10 years after birth whereas for others follow-up was truncated at 5 years. Considering the relatively high rates of ASD diagnoses in scholar age (Maenner et al. 2013), it is possible that some children were erroneously categorised as noncases because a diagnosis of ASD was made after 5 years of age. Clearly, it is difficult to speculate on whether this under recognition of cases might have somewhat hampered the analyses.

In conclusion, a possible link between prenatal exposure to SSRIs and development of ASDs, previously suggested by two case-control studies, was not confirmed by this very large cohort study which followed for 5-10 years more than 600000 births. However, this study failed to demonstrate that SSRI exposure during pregnancy is safe in terms of child development outcomes, as an increased risk of ASDs cannot be completely ruled out. On the basis of the upper boundary of the CI (ranging from 0.90 to 1.61), it cannot be excluded that SSRI exposure increases the relative risk of developing ASDs up to $61 \%$.

\section{Financial Support}

No financial support was received for this paper from any funding agency, commercial or not-for-profit sectors.

\section{Conflict of Interest}

None. 


\section{Ethical Standard}

The authors assert that all procedures contributing to this work comply with the ethical standards of the relevant national and institutional committees on human experimentation and with the Helsinki Declaration of 1975, as revised in 2008.

\section{References}

Barbui C, Gastaldon C, Cipriani A (2013). Benzodiazepines and risk of dementia: true association or reverse causation? Epidemiology and Psychiatric Sciences 22, 307-308.

Croen LA, Grether JK, Yoshida CK, Odouli R, Hendrick V (2011). Antidepressant use during pregnancy and childhood autism spectrum disorders. Archives of General Psychiatry 68, 1104-1112.

Grigoriadis S, VonderPorten EH, Mamisashvili L, Tomlinson G, Dennis CL, Koren G, Steiner M, Mousmanis P, Cheung A, Radford K, Martinovic J, Ross LE (2013). The impact of maternal depression during pregnancy on perinatal outcomes: a systematic review and meta-analysis. Journal of Clinical Psychiatry 74, 321-341.

Hviid A, Melbye M, Pasternak B (2013). Use of selective serotonin reuptake inhibitors during pregnancy and risk of autism. New England Journal of Medicine 369, 2406-2415.
Maenner MJ, Schieve LA, Rice CE, Cunniff C, Giarelli E, Kirby RS, Lee LC, Nicholas JS, Wingate MS, Durkin MS (2013).

Frequency and pattern of documented diagnostic features and the age of autism identification. Journal of the American Academy of Child and Adolescent Psychiatry 52, 401-413.

Neuhaus E, Beauchaine TP, Bernier R (2010).

Neurobiological correlates of social functioning in autism. Clinical Psychology Reviews 30, 733-748.

Rai D, Lee BK, Dalman C, Golding J, Lewis G, Magnusson C (2013). Parental depression, maternal antidepressant use during pregnancy, and risk of autism spectrum disorders: population based case-control study. British Medical Journal 346, f2059.

Rezendes DL, Scarpa A (2011). Associations between parental anxiety/depression and child behavior problems related to autism spectrum disorders: the roles of parenting stress and parenting self-Efficacy. Autism Research and Treatment, Article ID 395190. doi: 10.1155/2011/395190.

Ross LE, Grigoriadis S, Mamisashvili L, Vonderporten EH, Roerecke M, Rehm J, Dennis CL, Koren G, Steiner M, Mousmanis P, Cheung A (2013). Selected pregnancy and delivery outcomes after exposure to antidepressant medication: a systematic review and meta-analysis. JAMA Psychiatry 70, 436-443.

Salas M, Hofman A, Stricker BH (1999). Confounding by indication: an example of variation in the use of epidemiologic terminology. American Journal of Epidemiology 149, 981-983. 